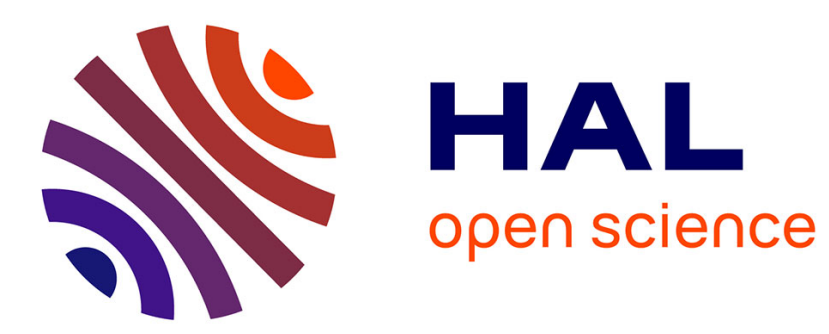

\title{
Numerical and analytical investigation of the indirect combustion noise in a nozzle
}

\author{
Matthieu Leyko, Franck Nicoud, Stéphane Moreau, Thierry Poinsot
}

\section{To cite this version:}

Matthieu Leyko, Franck Nicoud, Stéphane Moreau, Thierry Poinsot. Numerical and analytical investigation of the indirect combustion noise in a nozzle. Comptes rendus de l'Académie des sciences. Série IIb, Mécanique, 2009, 337 (6-7), pp.415-425. 10.1016/j.crme.2009.06.025 . hal-00803811

\section{HAL Id: hal-00803811 https://hal.science/hal-00803811}

Submitted on 22 Mar 2013

HAL is a multi-disciplinary open access archive for the deposit and dissemination of scientific research documents, whether they are published or not. The documents may come from teaching and research institutions in France or abroad, or from public or private research centers.
L'archive ouverte pluridisciplinaire HAL, est destinée au dépôt et à la diffusion de documents scientifiques de niveau recherche, publiés ou non, émanant des établissements d'enseignement et de recherche français ou étrangers, des laboratoires publics ou privés. 


\title{
Numerical and analytical investigation of the indirect noise in a nozzle
}

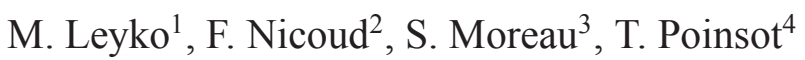

\begin{abstract}
Analytical and numerical assessments of the indirect noise generated through a nozzle are presented. The configuration corresponds to an experimental setup operated at DLR by Bake et al. (2008) where an entropy wave is generated upstream of the nozzle by means of an electrical heating device. Both 3D and 2D axisymmetric simulations are performed to demonstrate that the experiment is mostly driven by linear acoustic phenomena, including pressure wave reflection at the outlet and entropy-to-acoustic conversion in the accelerated regions. On the other hand, the spatial inhomogeneity of the upstream entropy fluctuation has no visible effect for the frequency range investigated $(0-100 \mathrm{~Hz})$. Results show that the acoustic impedance downstream of the nozzle must be accounted for appropriately in order to recover the experimental pressure signal. A good agreement is also obtained with a purely analytical assessment based on the compact nozzle approximation.
\end{abstract}

\section{INTRODUCTION}

Over the last five decades, jet and external aerodynamic noises of aircraft have been substantially reduced. Further developments will be needed for the modern aircraft design in order to meet the increasingly restrictive rules about noise reduction; there is no doubt that Computational AeroAcoustics (CAA) will play a major role in these future developments. With the drastic jet noise reduction already achieved, the relative importance of other noise sources has increased and the contribution of these sources must be controlled if further global noise reduction is to be achieved. Among these sources, the noise stemming from the turbulent flame within the combustor is already identified as non-negligible at take-off, especially in the mid frequency range. Two main mechanisms have been identified [13, 6, 14] regarding noise propagation from the combustion chamber to the far field (see Fig. 1):

- direct combustion noise: acoustic perturbations generated by the unsteady heat release from the turbulent flame [7] propagate either upstream or downstream through the turbomachinery stages and can reach the far field after it has been drastically distorted by the mean flow and also diffracted and reflected by the solid walls within the diffuser, the distributer, and the turbine and compressor blades.

- indirect combustion noise: entropy fluctuations generated within the combustion chambers (hot spots, imperfect mixing, ...) are propagated downstream and interact with accelerating mean flow. During this interaction, part of the energy contained in the entropy mode is transferred into the acoustic mode and the subsequent acoustic waves are transmitted to the far field through the turbine stages in a similar way as for the direct combustion noise.

From the current knowledge and expertise about Large Eddy Simulations (LES) of turbulent reacting flows in complex geometries and the accuracy requirements regarding noise prediction in CAA, computing the absolute level of combustion noise from a realistic combustor is certainly out of reach of the actual computing/modeling capabilities. The objectives of this study are three folds:

- to assess/establish the capability of a state-of-the art LES tool for the computation of compressible reacting flows to reproduce the entropy/acoustic interaction in the presence of a strong mean velocity gradient. To this end, the numerical results will be compared to the measurements from the entropy wave generator experiment studied at DLR by [4, 3, 2],

- to gain insight into the first order physical mechanisms that drive the pressure signal measured in the experiment. Notably the effect of a) the entropy fluctuations shape and size and b) the boundary conditions will be assessed,

\footnotetext{
${ }^{1}$ SNECMA, Villaroche, France - CERFACS, Toulouse, France

${ }^{2}$ Applied mathematics, University Montpellier II, France - franck.nicoud@univ-montp2.fr

${ }^{3}$ Valeo Engine Cooling, La Verrière, France

${ }^{4}$ CNRS IMF Toulouse, France
} 


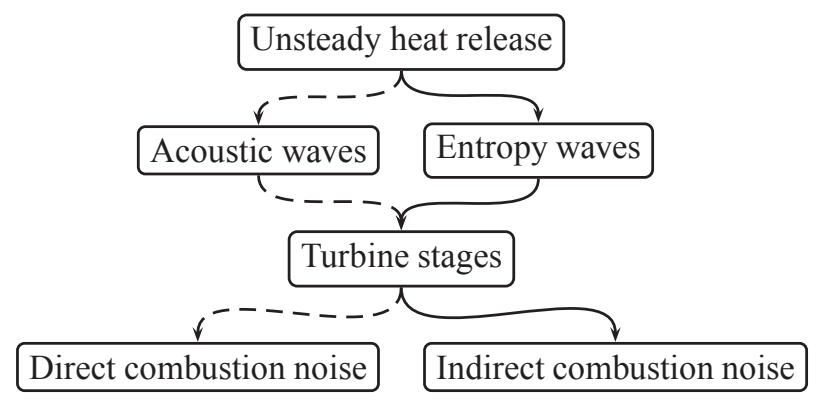

Figure 1 : The two main mechanisms for noise generation from confined flames: direct (--) and indirect (-) noise

- to investigate to what extent an analytical approach based on the compact nozzle approximation $[11,8]$ and valid for $1 \mathrm{D}$ planar waves can reproduce the experimental data of [4].

The analytical relationships that can be derived under the compact assumption for plane waves are first reviewed in section I. The experimental setup and associated computational domain are then discussed in section II where a short description of the LES solver is also provided. Finally, results are discussed in section III.

\section{THEORETICAL BACKGROUND}

This section builds upon the results obtained by [11] who focused on the generation and the transmission of flow perturbations (acoustic and entropy) through a steady non-uniform flow. Assuming a quasi-1D nozzle flow and quasi-steady perturbations (nozzle compactness), they established the relations linking the different perturbations using mass flow, energy and entropy conservations. These developments are reviewed here to provide a better understanding of the indirect noise generation mechanism.

\section{I.1 ISENTROPIC NOZZLE}

Consider an isentropic flow of a homogeneous gas of density $\rho$, velocity $u$, pressure $p$, constant heat capacity $c_{p}$ and $c_{v}=c_{p} / \gamma$ within a quasi-1D adiabatic duct of cross section area $\mathcal{A}(x)$. In this case, the mass flow

$$
\dot{m}=\rho u \mathcal{A},
$$

the total temperature

$$
T_{t}=T\left(1+\frac{\gamma-1}{2} \mathcal{M}^{2}\right)
$$

and the entropy

$$
s=c_{p} \log \frac{p}{\rho^{\gamma}}
$$

are constant throughout the duct. The above relations can be differentiated to yield:

$$
\begin{gathered}
\frac{d s}{c_{p}}=\frac{d p}{\gamma p}-\frac{d \rho}{\rho}, \\
\frac{d \dot{m}}{\dot{m}}=\frac{1}{\mathcal{M}} \frac{d u}{c}+\frac{d p}{\gamma p}-\frac{d s}{c_{p}}
\end{gathered}
$$

and

$$
\frac{d T_{t}}{T_{t}}=\frac{1}{1+\frac{\gamma-1}{2} \mathcal{M}^{2}}\left((\gamma-1) \mathcal{M} \frac{d u}{c}+(\gamma-1) \frac{d p}{\gamma p}+\frac{d s}{c_{p}}\right)
$$

where the speed of sound $c=\sqrt{\gamma p / \rho}$ and the Mach number $u / c$ have been introduced. Under the compact nozzle assumption (the wave lengths of the perturbations are large compared with the axial dimension of the nozzle), there is no delay and distortion between the inlet and the outlet of the nozzle. 


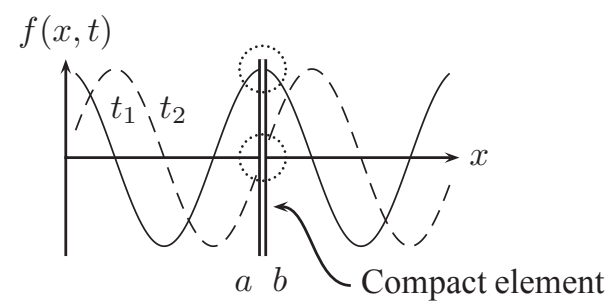

Figure 2 : Compact element illustration: The quantity $f$, conserved throughout the element, is the same upstream (a) and downstream (b) at each instant

As a result, the instantaneous values of the mass flow, the total temperature and the entropy are conserved throughout the nozzle at each instant, as displayed in Fig. 2. In other words, the compact nozzle assumption allows writing:

$$
[d s]_{a}^{b}=0 \quad ; \quad[d \dot{m}]_{a}^{b}=0 \quad ; \quad\left[d T_{t}\right]_{a}^{b}=0
$$

where []$_{a}^{b}$ stands for the jump between downstream (index $b$ ) and upstream (index $a$ ) of the related object (subcritical nozzle, supercritical nozzle and normal shock). At this point, it proves useful to introduce the following reduced variables and acoustic waves [11]:

$$
\nu=\frac{d u}{c} \quad ; \quad \varphi=\frac{d p}{\gamma p} \quad ; \quad \sigma=\frac{d s}{c_{p}} \quad ; \quad P^{+}=\frac{1}{2}(\varphi+\nu) \quad ; \quad P^{-}=\frac{1}{2}(\varphi-\nu)
$$

\section{I.1.1 UNCHOKED NOZZLE}

Combining Eq. 7 with Eqs.4, 5 and 6 the following set of three equations can be obtained:

$$
\begin{aligned}
-\left(1-\frac{1}{\mathcal{M}_{a}}\right) P_{a}^{-}+\left(1+\frac{1}{\mathcal{M}_{b}}\right) P_{b}^{+}-\sigma_{b} & = \\
-\left(1-\frac{1}{\mathcal{M}_{b}}\right) P_{b}^{-} & +\left(1+\frac{1}{\mathcal{M}_{a}}\right) P_{a}^{+}-\sigma_{a} \\
-\frac{(\gamma-1)\left(1-\mathcal{M}_{a}\right)}{1+\frac{\gamma-1}{2} \mathcal{M}_{a}^{2}} P_{a}^{-}+\frac{(\gamma-1)\left(1+\mathcal{M}_{b}\right)}{1+\frac{\gamma-1}{2} \mathcal{M}_{b}^{2}} P_{b}^{+}-\frac{1}{1+\frac{\gamma-1}{2} \mathcal{M}_{b}^{2}} \sigma_{b} & = \\
-\frac{(\gamma-1)\left(1-\mathcal{M}_{b}\right)}{1+\frac{\gamma-1}{2} \mathcal{M}_{b}^{2}} P_{b}^{-}+\frac{(\gamma-1)\left(1+\mathcal{M}_{a}\right)}{1+\frac{\gamma-1}{2} \mathcal{M}_{a}^{2}} P_{a}^{+} & -\frac{1}{1+\frac{\gamma-1}{2} \mathcal{M}_{a}^{2}} \sigma_{a} \\
\sigma_{b} & =\sigma_{a}
\end{aligned}
$$

In each of the previous equations, the LHS involves the three waves propagating outward, either upstream $\left(P_{a}^{-}\right)$or downstream $\left(P_{b}^{+}\right.$and $\left.\sigma_{b}\right)$; the RHS involves only (known) waves propagating inward, viz. towards the nozzle, either upstream $\left(P_{a}^{+}, \sigma_{a}\right)$ or downstream $\left(P_{b}^{-}\right)$. Note that in the particular case where $P_{a}^{+} \neq 0$, $\sigma_{a}=0$ and $P_{b}^{-}=0$ (the ingoing waves can be fixed freely), Eqs. 9 allows recovering the expression given in [11] for the acoustic response of the nozzle to an acoustic excitation (AA):

$$
\frac{P_{b}^{+}}{P_{a}^{+}}(\mathrm{AA})=\left(\frac{2 \mathcal{M}_{b}}{1+\mathcal{M}_{b}}\right)\left(\frac{1+\mathcal{M}_{a}}{\mathcal{M}_{a}+\mathcal{M}_{b}}\right)\left(\frac{1+\frac{1}{2}(\gamma-1) \mathcal{M}_{b}^{2}}{1+\frac{1}{2}(\gamma-1) \mathcal{M}_{a} \mathcal{M}_{b}}\right)
$$

In the same way, if one assumes $P_{a}^{+}=0, \sigma_{a} \neq 0$ and $P_{b}^{-}=0$, the acoustic response to an entropy perturbation (SA) is recovered as in [11], viz.:

$$
\frac{P_{b}^{+}}{\sigma_{a}}(\mathrm{SA})=\left(\frac{\mathcal{M}_{b}-\mathcal{M}_{a}}{1+\mathcal{M}_{b}}\right)\left(\frac{\frac{1}{2} \mathcal{M}_{b}}{1+\frac{1}{2}(\gamma-1) \mathcal{M}_{a} \mathcal{M}_{b}}\right)
$$

\section{I.1.2 CHOKED ISENTROPIC NOZZLE}

In the case of isentropic choked nozzle, the flow is subsonic in the convergent nozzle part and totally supersonic in the divergent nozzle part. In this case, the acoustic wave $P_{b}^{-}$leaves the domain and cannot be 
imposed anymore. Two waves enters the domain, namely $P_{a}^{+}$and $\sigma_{a}$, and four must be determined, namely three transmitted/generated waves $\left(P_{b}^{+}, P_{b}^{-}\right.$and $\left.\sigma_{b}\right)$ and one reflected/generated wave $\left(P_{a}^{-}\right)$. The critical mass flow equation is then introduced in order to close the problem $\left(\mathcal{A}^{\star}\right.$ being the critical section area and $\dot{m}^{\star}$ being the critical mass flow):

$$
\dot{m}^{\star}=\frac{p_{t 1}}{\sqrt{\gamma r T_{t 1}}} \mathcal{A}^{\star} \gamma\left(\frac{\gamma+1}{2}\right)^{-\frac{1}{2} \frac{\gamma+1}{\gamma-1}}
$$

Combining the differential of Eq. 12 with Eq. 5, one obtains the following additional condition:

$$
\frac{d u}{c}-\frac{\gamma-1}{2} \mathcal{M} \frac{d p}{\gamma p}-\frac{1}{2} \mathcal{M} \frac{d s}{c_{p}}=0
$$

which can be used to complete the previous jump equations 7 and close the system. After some algebra the following expressions for the four outgoing waves can be obtained:

$$
\begin{aligned}
\left(1-\frac{\gamma-1}{2} \mathcal{M}_{a}\right) P_{a}^{+}-\left(1+\frac{\gamma-1}{2} \mathcal{M}_{a}\right) P_{a}^{-}-\frac{1}{2} \mathcal{M}_{a} \sigma_{a} & =0 \\
\left(1-\frac{\gamma-1}{2} \mathcal{M}_{b}\right) P_{b}^{+}-\left(1+\frac{\gamma-1}{2} \mathcal{M}_{b}\right) P_{b}^{-}-\frac{1}{2} \mathcal{M}_{b} \sigma_{a} & =0 \\
P_{a}^{+}+P_{a}^{-} & =P_{b}^{+}+P_{b}^{-} \\
\sigma_{a} & =\sigma_{b}
\end{aligned}
$$

In many practical cases, however, a residual shock stands downstream of the throat of a choked nozzle and the mean flow is not isentropic anymore.

\section{I.2 WAVES FOR A SHOCK}

The interaction of acoustic, entropy or vorticity waves with a shock wave is a complicated problem [9, 10], but it can be managed quite well analytically in 1D [12]. By construction the flow immediately upstream and downstream of a normal shock is supersonic and subsonic respectively. This means that four waves are in-going in this case, namely $P_{a}^{+}, P_{a}^{-}, \sigma_{a}$ and $P_{b}^{-}$, while only two propagates in the outward direction, viz. $P_{b}^{+}$and $\sigma_{b}$. The derivation of the outgoing waves as a function of the ingoing ones builds upon the classical jump relations trough a normal shock which only depends on the upstream Mach number. This latter quantity is modified by the shock motion induced by the in-going waves. After some algebra, ones obtains:

$$
\begin{aligned}
\left(1+\mathcal{M}_{a}^{2}+2 \mathcal{M}_{a}^{2} \mathcal{M}_{b}\right) P_{b}^{+}+ & \left(1+\mathcal{M}_{a}^{2}-2 \mathcal{M}_{a}^{2} \mathcal{M}_{b}\right) P_{b}^{-} \\
\left(1+\mathcal{M}_{a}^{2}+2 \mathcal{M}_{a} \mathcal{M}_{b}^{2}\right) P_{a}^{+} & +\left(1+\mathcal{M}_{a}^{2}-2 \mathcal{M}_{a} \mathcal{M}_{b}^{2}\right) P_{a}^{-} \\
\left(P_{b}^{+}+P_{b}^{-}-P_{a}^{+}-P_{a}^{-}\right)(\gamma-1) \frac{\left(\mathcal{M}_{a}^{2}-1\right)^{2}}{\left((\gamma-1) \mathcal{M}_{a}^{2}+2\right) \mathcal{M}_{a}^{2}} & =\sigma_{b}-\sigma_{a}
\end{aligned}
$$

Eqs. 15 generalizes the result given in [11] to the case where $P_{b}^{-}$is not zero and indicates that entropy fluctuations can be generated by the interaction between an acoustic wave and a shock.

\section{Configuration}

All the computations presented in this paper are related to the DLR experimental setup studied by [2]. A sketch of the so-called Entropy Wave Generator experiment is displayed in Fig. 3. It consists in an accelerated tube fed by entropy waves generated by an electric heating device located between the upstream plenum and the nozzle. The operating conditions are such that the nozzle is choked but not adapted so that a normal shock takes place just after the throat, within the divergent section. When accelerated through the nozzle, the small amplitude entropy fluctuations (of order $10 \mathrm{~K}$ ) produce backward and forward propagating acoustic waves. The forward part of the generated noise is measured/analyzed by microphones located downstream the nozzle. The main physical parameters defining the operating conditions are gathered in Table 1. Note that the heating duration is $100 \mathrm{~ms}$ which, with a bulk velocity of order $12 \mathrm{~m} / \mathrm{s}$, leads to a entropy perturbation of typical length of order $1200 \mathrm{~mm}$ while the nozzle is about $263 \mathrm{~mm}$ long. This is 


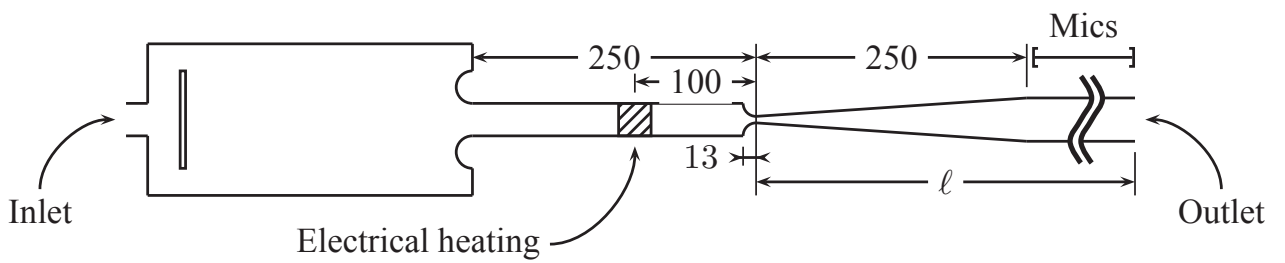

Figure 3 : Principle of the Entropy Wave Generator experiment (lengths are given in $\mathrm{mm}$ ). Short configuration: $\ell=$ $500 \mathrm{~mm}$; Long configuration: $\ell=2100 \mathrm{~mm}$

\begin{tabular}{|c|c|c|c|c|}
\hline Plenum pressure & Outlet pressure & Inlet Mach & Shock Mach & Outlet Mach \\
\hline $117000 \mathrm{~Pa}$ & $100800 \mathrm{~Pa}$ & 0.037 & 1.340 & 0.023 \\
\hline \hline Plenum temperature & Pulse amplitude & Pulse duration & Pulse length & \\
\hline $298 \mathrm{~K}$ & $9 \mathrm{~K}$ & $100 \mathrm{~ms}$ & $1000 \mathrm{~ms}$ & \\
\hline \hline Convergent length & Divergent length & Throat diameter & Inlet diameter & Outlet diameter \\
\hline $13 \mathrm{~mm}$ & $250 \mathrm{~mm}$ & $7.5 \mathrm{~mm}$ & $30 \mathrm{~mm}$ & $40 \mathrm{~mm}$ \\
\hline
\end{tabular}

Table 1 : Main characteristics of the experimental/numerical experiment

most probably not relevant to practical engine applications where the expected length of the entropy spots is more likely in the order $100-200 \mathrm{~mm}$.

The numerical tool used in this study is the unstructured combustion code AVBP developed at CERFACS [1]. AVBP solves the complete Navier-Stokes equations in two and three spatial dimensions. The unstructured approach allows computing not only the nozzle but also the whole air feeding line as well as the exhaust system. This code was selected because it solves the complete compressible Navier-Stokes equations and can naturally account for the energy transfer between the entropy and the acoustic modes of oscillation. The numerical method used is based on a weighted residual, Taylor-Galerkin discretization which is third order in both space and time [5] in order to minimize the dispersion/dissipation errors.

The main characteristics of the simulations performed are gathered in Table 2. The entries 'short' and 'long' refer to the two types of computational domain depicted in Fig. 3. They both include the upstream plenum and the heating section but the 'short' one extends only $500 \mathrm{~mm}$ downstream of the nozzle throat (length $\ell$ ) while the 'long' contains the exhaust duct until the entrance to the anechoic part, viz. approx. $2100 \mathrm{~mm}$ downstream of the nozzle. $\mathrm{BC}$ refers to the boundary condition prescribed at the outlet of the computational domain which can be either non-reflecting, fully reflecting or corresponds to a finite (neither zero nor infinite) acoustic impedance. The 'heating' entries correspond to the shape of the temperature pulse: it is 1D in most cases (viz. uniform in the plane normal to the duct) or it can depend on the distance $r$ to the axis (viz. non-uniform in the cross section - in the present case, the heating source term is proportional to $\cos (r / R \cdot \pi / 2)$, with $R$ the radius of the upstream duct). The uniform and non-uniform heatings correspond to the same overall energy so that the comparison between the runs $2 \mathrm{D}-1$ and $2 \mathrm{D}-2$ can provide relevant information regarding the effects of the inhomogeneity of the entropy perturbation on the noise generated. 


\begin{tabular}{|c|c|c|c|c|}
\hline Run & Geometry & Length & BC & Heating \\
\hline 3D-1 & $3 \mathrm{D}$ & short & non-reflecting & uniform \\
\hline $2 \mathrm{D}-1$ & $2 \mathrm{D}$ axi & short & non-reflecting & uniform \\
\hline 2D-2 & $2 \mathrm{D}$ axi & short & non-reflecting & non-uniform \\
\hline 2D-3 & $2 \mathrm{D}$ axi & short & reflecting & uniform \\
\hline 2D-4 & $2 \mathrm{D}$ axi & long & finite impedance & uniform \\
\hline
\end{tabular}

Table 2 : Main characteristics of the small scale simulations

In the same way, comparing runs 2D-1 and 2D-3 will tell about the sensitivity of the results to the outlet boundary condition while comparing 3D-1 and 2D-1 will be relevant to quantify the $3 \mathrm{D}$ effects in the observed results. At last, run 2D-4 is designed to mimic, as much as possible, the experimental downstream acoustic impedance 5 . In all cases, the mesh resolution is enough to represent the propagation of the entropy and acoustic waves in the duct without significant dissipative/dispersive errors (mesh size of order $1 \mathrm{~mm}$ compared to the perturbation size of order $1200 \mathrm{~mm}$ - see section $\S \mathrm{II})$. The 3D mesh is rather coarse with 1.2 million tetraedras, corresponding to approximately 15 cells in a cross section. The mesh density is equivalent for $2 \mathrm{D}$ axisymmetric cases. Note that the mesh refinement is not sufficient to resolve the residual shock appropriately which, given the non-dissipative character of the numerical method, can lead to numerical instabilities. Thus extra numerical dissipation has been added in both $3 \mathrm{D}$ and $2 \mathrm{D}$ axisymmetric calculation in the region downstream of the throat of the nozzle.

In order to mimic the experimental heating device, a source term is added to the energy equation. It reads:

$$
S(x, t)=S_{0} \cdot \frac{1}{2}\left[\tanh \left(\frac{x-x_{0}+L_{s} / 2}{a}\right) \cdot \tanh \left(-\frac{x-x_{0}-L_{s} / 2}{a}\right)+1\right] \cdot s(t)
$$

where $L_{s}=30 \mathrm{~mm}$ is representative of the length of the experimental heating zone and $a=3 \mathrm{~mm}$ enables to smooth sufficiently the source term to avoid numerical issues. Moreover, the average location $x_{0}$ of the source term has been consistently chosen with the location of the electrical device in the experiment (100 $\mathrm{mm}$ upstream of the nozzle throat). The temporal evolution $s(t)$ is defined as following:

$$
s(t)= \begin{cases}1-e^{-\frac{t-t_{0}}{\tau}} & \text { if } t \in\left[t_{0}, t_{0}+T\right] \\ s\left(t_{0}+T\right) e^{-\frac{t-t_{0}}{\tau}} & \text { if } t>t_{0}+T\end{cases}
$$

where $t_{0}$ is the time when the electrical device is triggered, $T$ is the pulse duration and is equal to $100 \mathrm{~ms}$ and $\tau=8 \mathrm{~ms}$. As shown in Fig 4, these numerical parameters allows a fair representation of the temperature fluctuation produced in the experiment. The next section analyzes the results obtained in terms of pressure fluctuations generated downstream when the temperature perturbation displayed in Fig. 4 passes through the nozzle.

\section{RESULTS}

The EWG setup has been studied both numerically and analytically during this study. Numerical simulations have the advantage to integrate all the possible effects involved in the entropy noise generation and

\footnotetext{
${ }^{5}$ not available in $[4,3,2]$ but kindly provided by Dr. Ing. F. Bake during the course of this study
} 


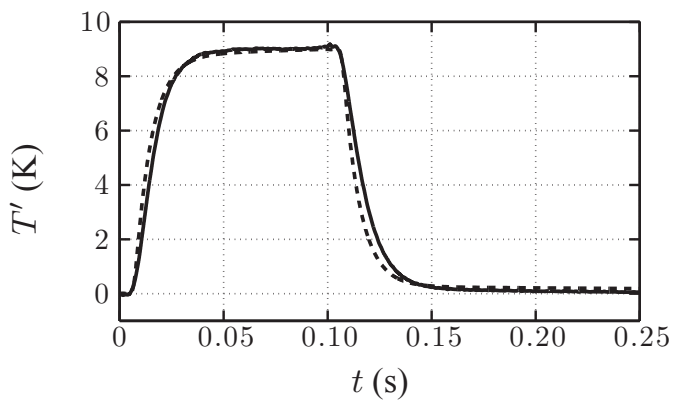

Figure 4 : Time traces of the experimental (-) and numerical (--) temperature downstream of the heating location
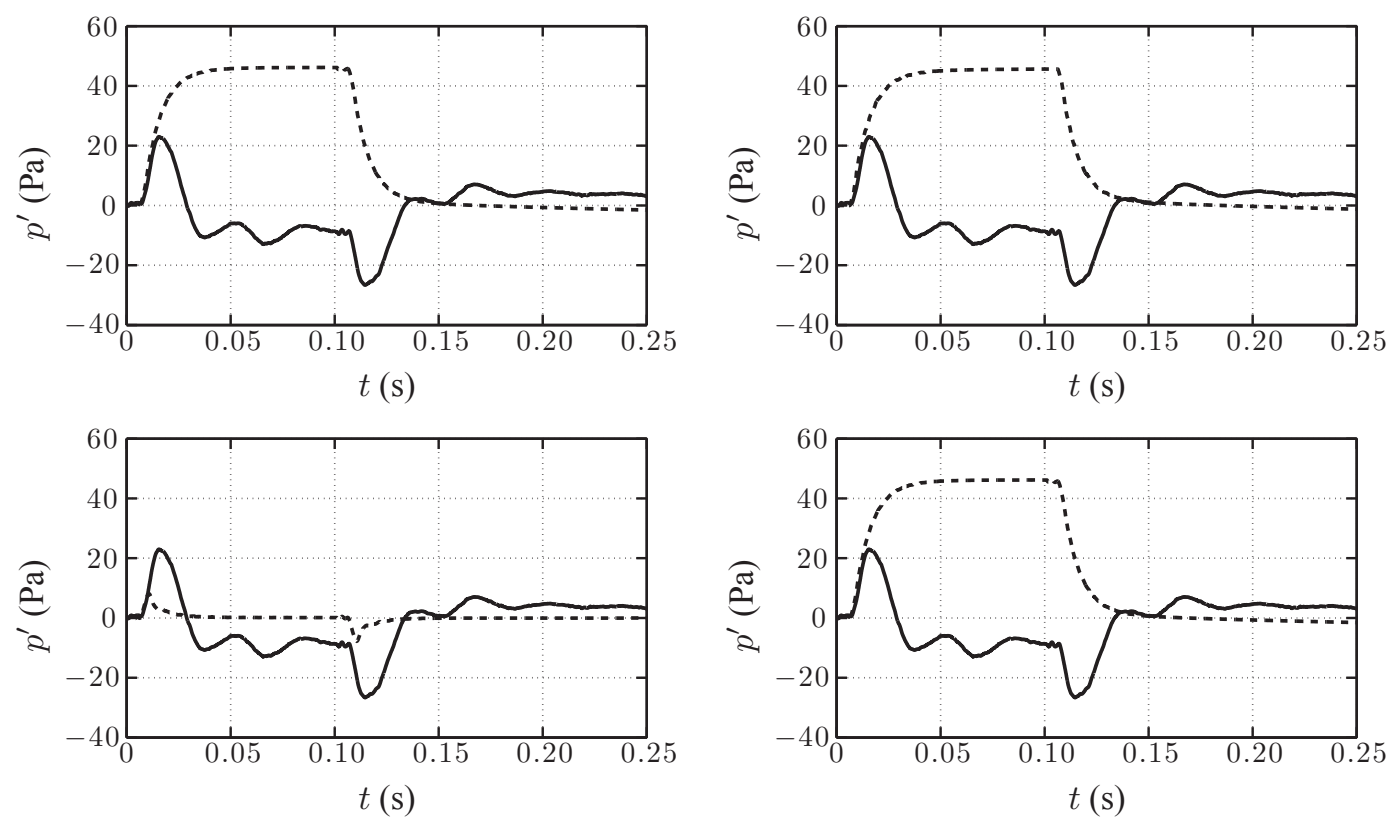

Figure 5 : Time traces of the fluctuating pressure $350 \mathrm{~mm}$ downstream of the nozzle. Experimental data: -; Numerical result: -- Top left: run 2D-1; Top right: run 2D-2; Bottom left: run 2D-3; Bottom right: run $3 D-1$

contained in the Navier-Stokes equations. The analytical approach is valid only under the compact nozzle assumption and in the linear regime.

The time traces of the pressure computed $350 \mathrm{~mm}$ downstream of the throat are displayed in Fig. 5 for runs 2D-1, 2D-2, 2D-3 and 3D-1 together with the experimental signal. Clearly enough, these simulations do not reproduce the experimental data, neither in terms of amplitude, nor in terms of signal shape. For runs 2D-1 and 3D-1, the numerical pressure trace has a top hat behavior similar to the temperature upstream fluctuation while the experiment shows a wavy behavior at a frequency close to $30 \mathrm{~Hz}$. The figure also indicates that $3 \mathrm{D}-1$ leads to results very similar to $2 \mathrm{D}-1$, indicating that the disagreement between the $2 \mathrm{D}-1$ computation and the experimental data cannot be attributed to 3D effects. The same conclusion can be drawn for the temperature inhomogeneity which appears to have no effect, at least for the configuration considered (comparing 2D-1 and 2D-2). On the other hand, Fig. 5 illustrates how large the effects of the downstream acoustic boundary condition can be. When a fully reflecting condition (which imposes pressure) is used instead of a non-reflecting one (which essentially set the incoming acoustic wave to zero at the outlet), the amplitude decreases drastically. More importantly, the shape of the signal is also strongly modified by the superposition of the downward and backward pressure waves. A wavy behavior is obtained with run 2D-3, although with characteristic amplitude and frequency in quantitative disagreement with the experiment. The previous results suggest that the discrepancies observed in Fig. 5 might be related to a wrong downstream acoustic impedance. Indeed, the short computational domain and non-reflecting BC 

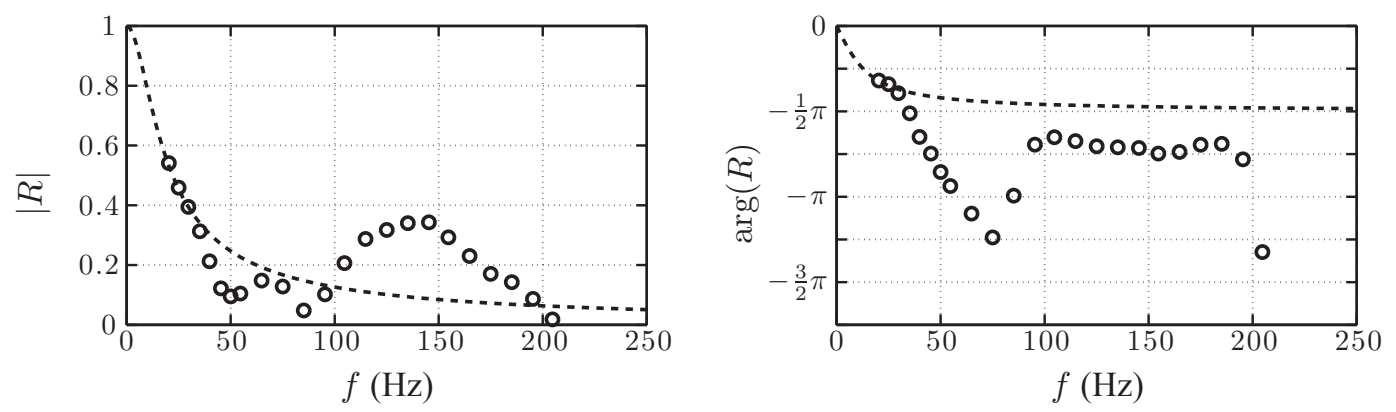

Figure 6 : Experimental reflection coefficient shifted $2100 \mathrm{~mm}$ downstream of the nozzle throat and numerical reflection coefficient. Shifted experimental data: $\circ$; Tuned relaxation coefficient: --

used in run 2D-1, 2D-2 and 3D-1 would only be representative of the actual experimental conditions if a perfect anechoic system would have been used by [2]. Actually, Fig. 6 demonstrates that this is not the case and that substantial reflection occurs especially in the low frequency range: the modulus of the reflection coefficient, defined as the ratio of the backward wave to forward wave at the outlet, is as large as 0.5 at 30 $\mathrm{Hz}$, the typical frequency of the reflections observed in the experimental signal (see Fig. 5). Accounting for a complex valued, frequency dependent reflection coefficient in a CFD code solving the flow equations in the time domain is not an easy task although it can be theoretically done by making use of recursive/nonrecursive digital filters. A simpler approach has been followed in the present study. Instead of using a non-reflecting outlet boundary condition with zero entering wave $L^{-}$, it is common use to write the latter as a pressure difference times a relaxation coefficient $\kappa[15]$, viz. $L^{-}=2 \kappa \Delta t \cdot\left(p_{\text {ref }}-p_{B}\right) /(\rho c)$, with $\Delta t$ the time step, $p_{B}$ the nodal pressure at the outlet boundary and $p_{\text {ref }}$ the reference pressure. In doing so, the outlet condition acts as a first order low pass filter [16] whose cut-off frequency is inversely proportional to $\kappa$ and the reflection coefficient $R$ reads:

$$
R=-\frac{1}{2 \imath \omega / \kappa+1}
$$

It is thus possible to tune the relaxation coefficient $\kappa$ in order to mimic the amplitude of the experimental reflection coefficient, at least in the low frequency range. The length $\ell$ of the downstream duct is then tuned in such a way to mimic the experimental time delay as well as to compensate for the phase of the relaxation-based outlet partially reflecting condition. The experimental reflection coefficient is considered here to be measured at the nozzle throat $(x=0)$ while the reflection coefficient of numerical boundary condition (Eq. 16) is given at the end of the computational domain $(x=\ell)$. The experimental reflection coefficient is thus multiplied by $\exp (-2 \imath \ell \omega / c)$ in order to shift it to the same position, at the end of the numerical domain $(x=\ell)$. The experimental reflection coefficient obtained in this way is depicted in Fig. 6 where a fair agreement with the numerical one is apparent in the frequency range $20-40 \mathrm{~Hz}$. The 'optimized' relaxation coefficient $\kappa$ and length $\ell$ are close to $160 \mathrm{~s}^{-1}$ and $2100 \mathrm{~mm}$. As anticipated, Fig. 7 indicates that the numerical pressure signal is in better agreement with the measured one when accounting more accurately for the effective downstream boundary condition.

It is also possible to perform all these calculations analytically. This can be done using the relations presented previously for the supercritical nozzle (Eq. 14), the normal shock (Eq. 15) and the subcritical nozzle (Eq. 9). The supercritical nozzle starts at point 1 at the nozzle inlet and finishes at point 2 in the divergent section where the normal shock takes place. The normal shock relations are defined between point 2 and 3 and finally the subcritical nozzle starts at point 3, downstream of the shock, and finishes at point 4 at the end of the divergent section as shown Fig. 8. Only the entropy wave generated by the electrical device is taken into account and the upstream part of the nozzle is assumed to be non-reflecting (even if this is not the case in the real configuration). The subsonic nozzle outlet is represented in a general manner by the reflection coefficient $R=P_{4}^{-} / P_{4}^{+}$, but it will be considered to be infinitely small for the non-reflecting calculation ( $R \ll 1$ for numerical issues) and to be equal to the tuned reflection coefficient of the numerical computation 2D-4 for the 'real' case. The entropy wave temporal evolution is proportional to the one imposed on the source term on energy in the numerical computation. All the analytical calculations are performed in the frequency domain in order to take into account the frequency dependence of the outlet 

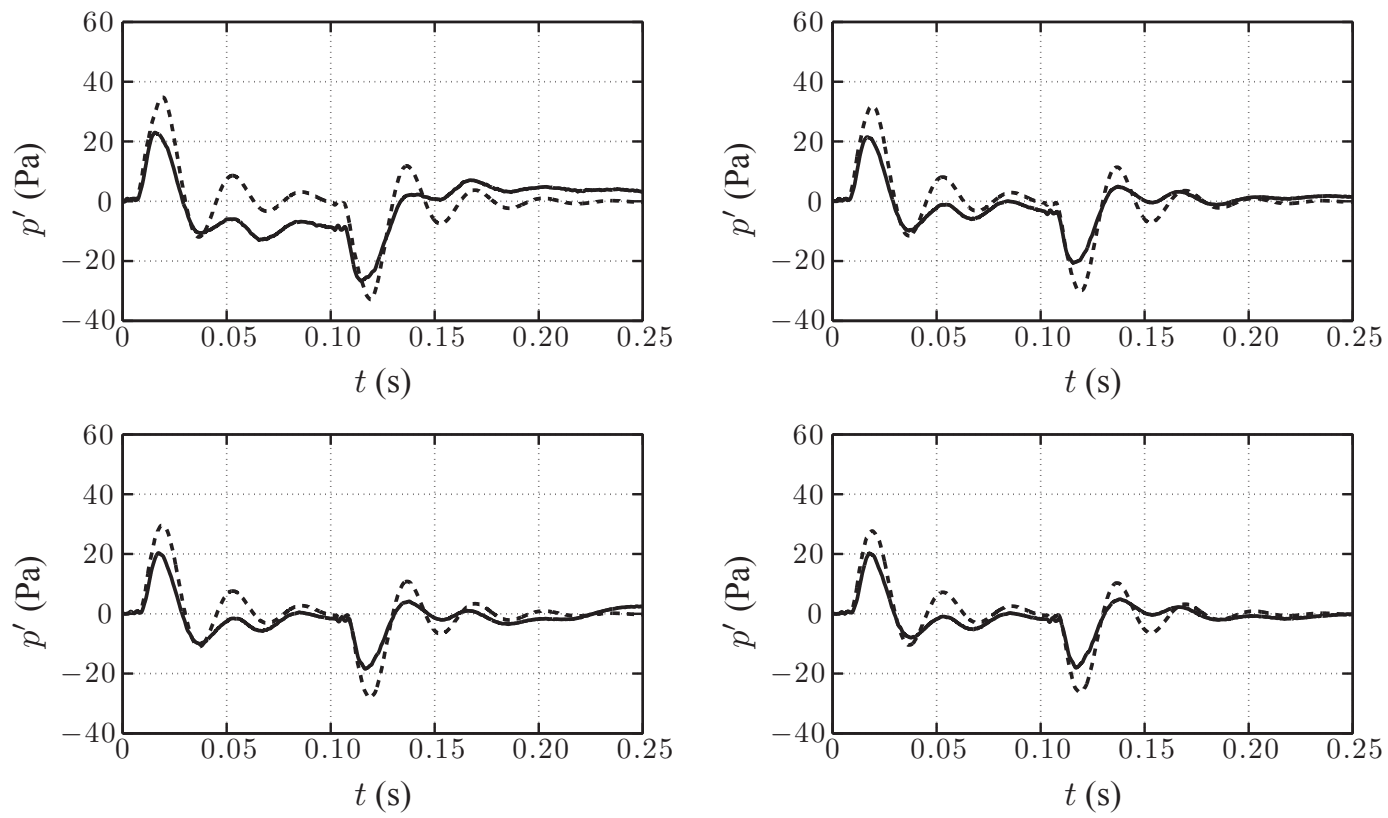

Figure 7 : Time traces of the fluctuating pressure downstream of the nozzle. Experimental data: -; Run 2D-4: --. Distance downstream of the nozzle : Top left: $350 \mathrm{~mm}$; Top right: $730 \mathrm{~mm}$; Bottom left: $975 \mathrm{~mm}$; Bottom right: $1150 \mathrm{~mm}$

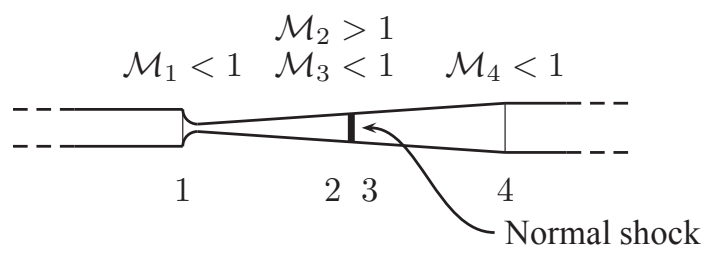

Figure 8 : Description of the analytic approach

boundary condition in the real case, but the different nozzle elements are still assumed to be compact. Since the case of a shock in the divergent section is considered here, the supercritical nozzle (from 1 to 2) can be treated independently of the downstream elements, and provide explicitly the waves entering the downstream elements. The shock (from 2 to 3 ) and the subcritical nozzle with the outlet BC (from 3 to 4 ) have to be treated together since most of the waves are coupled. Finally, one obtains the reduced pressure fluctuation $\varphi_{4}(\omega)$ in the frequency domain as a function of the four mach numbers $\mathcal{M}_{1}, \mathcal{M}_{2}, \mathcal{M}_{3}$ and $\mathcal{M}_{4}\left(\mathcal{M}_{3}\right.$ and $\mathcal{M}_{4}$ are actually linked by the shock relations), the reflection coefficient $R(\omega)$ and the reduced entropy fluctuation $\sigma_{1}(\omega)$. The time evolutions of the pressure fluctuations reconstructed from the analytical calculations are compared to the numerical computations. Fig. 9 shows a very good agreement between these two approaches. This demonstrates that the EWG experiment (for the given regime) is essentially driven by linear quasi-1D acoustics.

\section{Conclusions}

The numerical and analytical analysis performed in this study demonstrates that the pressure signals obtained in the EWG experiment by [2] result from two main mechanisms:

- the entropy-to-acoustic conversion due the strong mean velocity gradient in the nozzle, including the normal shock that stands just downstream of the throat,

- the acoustic reflection within the exhaust system downstream of the nozzle and test section. 

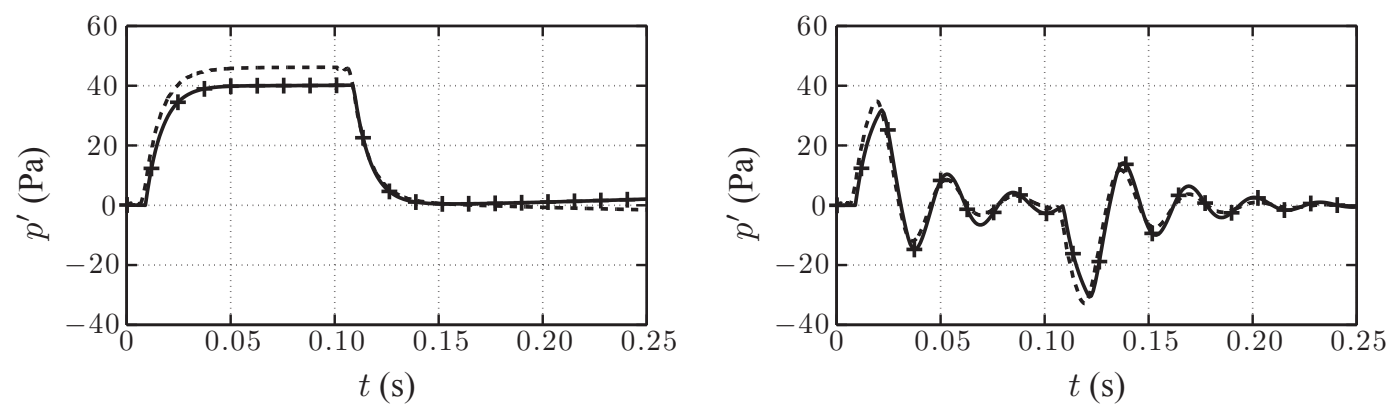

Figure 9 : Time traces of the fluctuating pressure downstream of the nozzle. Numerical results: --; Analytical results: -+- . Left: Non-reflecting case (Analytic compared to Run 2D-1); Right: Finite impedance case (Analytic compared to Run $2 D-4)$.

Moreover, in the low frequency range investigated, only 1D planar waves are present and the compact nozzle approximation is valid, even for the entropy perturbations. As a result, the pressure signals observed experimentally and numerically can be nicely reproduced by a simple quasi-1D analytical model derived in the zero frequency limit for the nozzle.

The authors gratefully acknowledge support from CINES for the computer ressources and the DLR for providing the experimental data.

\section{REFERENCES}

[1] AVBP. Avbp code: www.cerfacs.fr/cfd/avbp_code.php and www.cerfacs.fr/cfd/cfdpublications.html, 2008.

[2] F. Bake, N. Kings, and I. Rohle. Fundamental mechanism of entropy noise in aero-engines: experimental investigation. J. Eng. Gas Turb. and Power, 130, 2008.

[3] F. Bake, U. Michel, and I. Rohle. Investigation of entropy noise in aero-engine combustors. In Transaction of the ASME - 129, 2007.

[4] F. Bake, U. Michel, I. Rohle, C. Richter, F. Thiele, M. Liu, and B. Noll. Indirect combustion noise generation in gas turbines. In 11th AIAA/CEAS Aeroacoustics Conference - AIAA 2005-2830, 2005.

[5] O. Colin and M. Rudgyard. Development of high-order taylor-galerkin schemes for unsteady calculations. J. Comput. Phys., 162(2):338-371, 2000.

[6] N.A. Cumpsty and F.E. Marble. The interaction of entropy fluctuations with turbine blade rows ; a mechanism of turbojet engine noise. Proc. R. Soc. Lond., 357:323-344, 1977.

[7] M. Ihme, H. Pitsch, and D. Bodony. Radiation of noise in turbulent non-premixed flames. In Proc. of the Combustion Institute, 2008.

[8] M. Leyko, F. Nicoud, and T. Poinsot. Comparison of indirect and direct combustion noise in aircraft engines. In 11th CEAS-ASC Workshop of X3-Noise, 2007.

[9] K. Mahesh, S. Lee, S.K. Lele, and P. Moin. The interaction of an isotropic field of acoustic waves with a shock wave. J. Fluid Mech., 300:383-407, 1995.

[10] K. Mahesh, S.K. Lele, and P. Moin. The influence of entropy fluctuations on the interaction of turbulence with a shock. J. Fluid Mech., 334:353-379, 1997.

[11] F.E. Marble and S. Candel. Acoustic disturbances from gas nonuniformities convected through a nozzle. J. Sound Vibration, 55:225-243, 1977.

[12] W.H. Moase, M.J. Brear, and C. Manzie. The forced response of choked nozzles and supersonic diffusers. J. Fluid Mech., 585:281-304, 2007. 
[13] M. Muthukrishnan, W.C. Strahle, and D.H. Neale. Separation of hydrodynamic, entropy, and combustion noise in a gas turbine combustor. AIAA Journal, 16(4):320-327, 1978.

[14] G. F. Pickett. Core engine noise due to temperature fluctuations convecting through turbine blade rows. In 2nd AIAA Aeroacoustics Conference - AIAA 1975-528, 1975.

[15] T. Poinsot and S. Lele. Boundary conditions for direct simulations of compressible viscous flows. J. Comput. Phys., 101(1):104-129, 1992.

[16] L. Selle, F. Nicoud, and T. Poinsot. The actual impedance of non-reflecting boundary conditions: implications for the computation of resonators. AIAA Journal, 42(5):958-964, 2004. 